\title{
LA CONTINUIDAD DEL CRECIMIENTO ECONÓMICO EN ESPAÑA. 1850-1936*
}

\author{
ANTONIO CUBEL MONTESINOS \\ y JORDI PALAFOX GAMIR \\ Universidad de Valencia
}

\section{RESUMEN}

El crecimiento de la economía española durante los siglos $\mathrm{xx}$ y $\mathrm{xx}$ ha sido en gran medida singular. Mientras en el siglo XIX España permaneció al margen del proceso de industrialización, durante el siglo $\mathrm{xx}$ se ha unido al reducido grupo de los países desarrollados. Este artículo contrasta para la etapa 1850-1936 la existencia de rupturas dentro de este proceso, aplicando diferentes tests econométricos asociados a los trabajos de Perron y de Zivot y Andrews, a las series de PNB, producción industrial e inversión. Los resultados confirman una continuidad básica en el crecimiento español. Sin embargo, muestran también la existencia de dos cambios de nivel: 1870 para la producción industrial y 1919 en la inversión pública

\section{ABSTRACT}

The Spanish pattern of economic growth during the last two centuries is quite unique. In the nineteenth century the country remained outside the process of industrialization, but during the twentieth century he has joined the small group of developed economies. This article tests the existence of

* Estas páginas forman parte de una investigación más amplia financiada con una ayuda interna del Instituo Valenciano de Investigaciones Económicas para el año 1996. Queremos agradecer a dos evaluadores anónimos, a José García Montalvo y a Joaquín Maudos, ambos investigadores del IVIE y profesores de Análisis Económico de la Universidad de Valencia, sus comentarios sobre la sección tercera relacionada con los contrastes econométricos realizados. Como es obvio, no son responsables de las tesis mantenidas ni de las deficiencias que puedan subsistir. A Leandro Prados de la Escosura no podemos dejar de agradecerle su generosidad al facilitarnos su estimación provisional del PNB. 
discontinuities between 1850-1936 in the series of PNB, industrial production and private and public investment by utilizing recent developments in the econometric analysis associated with the work of Perron and Zivot and Andrews. The results confirm the continuity of the Spanish growth during the decades considered. However, they also show two breakpoint years: 1870 in the serie of industrial production and 1919 in the data of public investment.

\section{INTRODUCCIÓN}

El crecimiento económico español durante los dos últimos siglos ha sido, sin duda, un proceso singular. Como ha sintetizado Tortella: «Si hay acuerdo entre los autores de que el siglo XIX fue el del fracaso, también lo hay acerca de que el xx fue el del éxito de la industrialización» ${ }^{1}$. De esta forma, durante la centuria en que otras economías europeas experimentaron, tras Gran Bretaña, un profundo proceso de cambio estructural que consolidó en ellas la industrialización, la economía española mantuvo la preponderancia del sector agrario, hasta el extremo de que en 1900, la población activa del sector primario representaba dos tercios del total ${ }^{2}$. Por contra, a lo largo del siglo actual, durante el cual la industrialización ha sido un resultado excepcional del crecimiento económico, España ha conseguido transformar radicalmente la estructura de su economía incorporándose al limitado grupo de países desarrollados. Las consecuencias políticas de la Segunda Guerra Mundial y sus repercusiones sobre las economías del centro y este de Europa, y en especial sobre aquellas que en los años previos al conflicto habían alcanzado un nivel de renta por habitante más elevado, invitan a no exagerar esta excepcionalidad. Pero ello no puede llevar a ignorar que las transformaciones consolidadas durante los años cincuenta y sesenta del siglo actual constituyen un resultado poco frecuente en la historia de la economía del siglo xx.

Uno de los interrogantes suscitados por esta evolución es identificar cuál es la trascendencia de su trayectoria hasta 1936 en los cambios ocurridos durante el segundo tercio del siglo. Responder a esta cuestión constituye uno de los retos colectivos de la investigación sobre el crecimiento económico de España en el largo plazo y sobrepasa, como es obvio, el objetivo de estas páginas. Sin embargo, los notables avances

\footnotetext{
1 Tortella (1994), p. 255.

${ }^{2}$ Nicolau (1989), en Carreras, coord. (1989), p. 79.
} 
realizados por la historia económica cuantitativa española y las posibilidades que brindan los avances estadístico-econométricos, sí hacen posible aportar nueva información sobre un aspecto relevante de este marco general retiradamente abordado en la historiografía: cen qué medida la consolidación de la sociedad industrializada en España ha sido un proceso de cambio estructural de largo plazo cuyo inicio puede fijarse en fechas muy anteriores a su culminación o ha sido, por contra, el resultado de un lento pero sostenido aumento del producto hasta alcanzar el umbral suficiente para provocar, dentro de la favorable coyuntura internacional de los años cincuenta y sesenta, un rápido proceso de transformaciones estructurales?

Como es fácil advertir, la pregunta se relaciona directamente con un debate de larga tradición en la historiografía tanto española como de otros países y muy especialmente en la británica: las continuidades y rupturas del proceso de industrialización. Aun cuando fue durante los años sesenta y setenta cuando el debate sobre esta disyuntiva alcanzó su apogeo [Gerschenkron (1968), pp. 11-39 y 77-97, Rostow (1960, 1963), Fishlow (1965) y Kuznets (1963)], en años recientes se han publicado también aportaciones relevantes sobre los rasgos dominantes de los procesos de crecimiento que acompañan a la industrialización ${ }^{3}$. El caso de España no ha sido una excepción. El influyente libro de Jordi Nadal (1975) planteó con rotundidad el fracaso de su industrialización durante el largo siglo xIX que transcurre entre 1814 y 1913, del - como indicaba su subtítulomodelo clásico, a la inglesa, ante las limitaciones de la demanda interna $\mathrm{y}$ a pesar de sus prometedores inicios. Una tesis ampliamente debatida, a la cual diversos investigadores, entre los que destaca Antonio Gómez Mendoza (1995, 1997), han realizado matizaciones muy destacadas insistiendo en los condicionantes naturales. Por su parte, partiendo de su monumental investigación cuantitativa de comienzos de los años ochenta, Albert Carreras (1990) ha ofrecido sugerentes precisiones sobre las pautas del crecimiento económico español desde 1850 y Leandro Prados de la Escosura (1995) viene, desde hace años, continuando esta labor fundamental de cuantificación del crecimiento contemporáneo con una estimación del PNB que, aun cuando todavía no finalizada, ha sido utilizado por diferentes investigadores ${ }^{4}$.

${ }^{3}$ Tanto en el terreno histórico como en el teórico, en donde una parte de las aportaciones recientes parten explícitamente del debate de los años sesenta. A título de ejemplo: Murphy, Shleifer y Vishny (1989), pp. 1003-1004, y Aranzadis y Drazen (1990), pp. 501-503.

4 A título de ejemplo: Herranz y Tirado (1996), pp. 11-49, y Tirado (1996), en especial cap. 9 , pp. 305 ss. 
Dentro de este contexto, estas páginas pretenden aportar información sobre los rasgos básicos del crecimiento español, contrastando la existencia de discontinuidades estadísticamente significativas en la trayectoria de la economía española en el largo período transcurrido entre 1850 y 1936. Su intención implícita es contribuir a confirmar o rechazar la existencia de una etapa de alteración profunda en las pautas de crecimiento previas a la consolidación de la sociedad industrializada en la segunda mitad del siglo $\mathrm{xx}$, reflejada bien directamente en el producto, bien indirectamente en la inversión. Entronca, pues, con varios de los debates recientes de la historia económica española, como la posible discontinuidad en la evolución entre las dos centurias o la identificación de coyunturas particularmente relevantes en el crecimiento a largo plazo en relación con la política arancelaria y más en general el sector exterior o con el cambio tecnológico en los primeros decenios del siglo $\mathrm{xx}$ en gran medida vinculado a la electrificación.

El texto está organizado de una forma muy sencilla. A continuación, en una primera sección se presenta con un nivel de detalle elevado la metodología utilizada, por cuanto hasta ahora no ha sido aplicada en la historia económica española. Tras ello, en el cuerpo central del artículo, se exponen consecutivamente los resultados obtenidos de su aplicación a la serie de $\mathrm{PNB}$, de producción industrial y de inversión, tanto privada como pública ${ }^{5}$, mientras las representaciones gráficas de los tests realizados forman el Apéndice incluido al final. Se ha optado por esta exposición secuencial frente a la posibilidad de un comentario conjunto de resultados, dado el objetivo del artículo ya señalado y, sobre todo, porque los debates historiográficos han tenido lugar en gran medida en relación con la tendencia mostrada por cada una de las series separadamente. Finalmente se incluye una brevísima recapitulación de los principales resultados.

A pesar del carácter econométrico de los tests utilizados, el texto quiere ser una contribución de historia económica. En nuestra opinión, la utilización de este tipo de técnicas permite hacer más explícitas algunas de las controversias existentes, aportando al mismo tiempo evidencia cuantitativa para alcanzar conclusiones más sólidas. Indudablemente, su empleo, al igual que las conclusiones a obtener, debe ser cauteloso, al

${ }^{5}$ PNB: Prados de la Escosura (1995); IPIES: Carreras (1984); Inversión: Cubel y Palafox (1997) e Inversión Pública: Pérez, Mas y Uriel (1996). Se ha preferido el uso de esta última serie frente a la del Instituo de Estudios Fiscales (1976) por estar elaborada con los criterios de Contabilidad Nacional, lo cual implica incluir las inversiones en mantenimiento. Ello hace aumentar sensiblemente los valores de la inversión pública. 
estar basadas en la elaboración de series siempre susceptibles de revisión y mejora. Nuestro objetivo al emplearlas no es otro que sugerir caminos nuevos, o complementarios, de interpretación de las transformaciones de la economía española en el largo plazo.

\section{LA ESTIMACIÓN DE UNA RUPTURA EN EL COMPORTAMIENTO DE SERIES MACROECONÓMICAS}

El concepto de cambio estructural en economía puede tener distintos significados. En su acepción más estricta se refiere a cambios en la estructura del sistema económico que afectan su forma de funcionamiento, como puede ser la transición del feudalismo al capitalismo o el paso de una economía de mercado a una de planificación central. En un sentido más laxo podemos referinos a cambios en el comportamiento de las variables provocados por medidas de política económica o shocks macroeconómicos. Es en este segundo sentido, para el que suele utilizarse con preferencia el término discontinuidades, en el que emplearemos el concepto.

Su medición en historia económica se ha beneficiado en los últimos años de los avances en la literatura estadística y económica sobre la modelización del comportamiento de series macroeconómicas y especialmente sobre existencia de raíces unitarias. La utilización de estos contrastes ha aportado nuevos elementos al debate sobre la existencia de una ruptura en la tendencia de la producción industrial en Gran Bretaña en torno a 1780 . Su intención, como es obvio, ha sido contrastar en qué medida el período 1780-1851 supuso una alteración estructural tal y como se ha venido caracterizando a la Revolución Industrial ${ }^{6}$. También se han analizado las discontinuidades en la serie de la producción industrial como consecuencia de la Primera Guerra Mundial con resultados novedosos respecto a la influencia de la vuelta a la paridad de 1925 y las repercusiones de la crisis de $1929^{7}$.

${ }^{6}$ Crafts y Harley (1992), pp. 703-730; Crafts y Mill (1994), pp. 769-775, y Greasley y Oaxley (1994), pp. 766-768. Un repaso de su aplicación fuera de la historia económica en Maddala y Kim (1996), pp. 73-103.

7 Greasley y Oaxley (1996), pp. 82-100. Un contraste, en gran parte similar, ha sido aplicado por Inwood y Stengos (1991), pp. 274-286, a la trayectoria de la economía canadiense entre 1870 y 1985 . Puede verse también la aplicación de la técnica de las trayectorias segmentadas en Crafts y Mills (1996), pp. 415-431. 
El origen analítico de estos métodos puede vincularse a un artículo de Perron (1989), en el que eran utilizados para analizar la influencia de dos momentos de ruptura en un conjunto de series macroecónomicas caracterizadas por la presencia de raíces unitarias: el crack de 1929 y la crisis del petróleo de 1973. A pesar de la influencia de su aportación, el uso de información exógena a la serie para determinar el momento de la ruptura fue criticado por la posibilidad de que sesgase el resultado hacia la aceptación de la hipótesis de cambio planteada. Para evitarlo, se ha sugerido realizar estimaciones de forma secuencial hasta determinar de forma endógena el momento temporal en el que la existencia del cambio resultara más probable y contrastar el resultado obtenido con nuevos test más exigentes. Éste es el método empleado en este artículo.

El comportamiento de una serie temporal, $\boldsymbol{y}_{t}$, se puede representar como la suma de una tendencia y un componente aleatorio:

$$
y_{t}=T D_{t}+Z_{t}
$$

donde $\mathrm{TD}_{\mathrm{t}}$ es una tendencia determinista y $Z_{\mathrm{t}}$ es el componente estocástico de $y_{t}$. Se puede emplear una amplia variedad de especificaciones para $T_{t}$, por ejemplo, $\mathrm{TD}_{\mathrm{t}}=0$, una constante, o una tendencia lineal o no lineal. En los contrastes realizados y para homogeneizar los resultados con otras aplicaciones dentro de la historia económica se ha empleado una tendencia lineal en tiempo:

$$
T D_{t}=\mu+\beta t
$$

de acuerdo con la cual el comportamiento de cualquier variable económica, por ejemplo el PNB, se puede caracterizar por:

$$
y_{t}=\mu+\beta t+u_{t}
$$

donde el nivel del producto en un momento $t$ es el resultado de un nivel inicial, $\mu$, el efecto acumulado del crecimiento de períodos anteriores representado por la tendencia $B t$, y un elemento estocástico, $u_{t}$, que refleja cualquier desviación de la senda del crecimiento lineal definido de forma determinista por $\mu, \boldsymbol{B}_{\mathrm{y}} \boldsymbol{t}$.

En la última década esta forma de representar las series ha sido puesto en duda. Los análisis de Nelson y Plosser (1982) demostraron que la mayoría de las variables macroeconómicas se caracterizan por la presencia de una raíz unitaria. La hipótesis de la raíz unitaria afecta al componente estocástico. En lugar de comportarse como un ruido blanco, es decir, con media cero y varianza constante a lo largo del tiempo, en presencia de 
una raíz unitaria se puede descomponer en un componente estocástico que afecta a la constante de la tendencia, $\mu$, en cada período y en un componente cíclico sin efectos a largo plazo sobre el nivel de $Z_{t}$, es decir, con media y varianza incondicionales a lo largo del tiempo. Estas series son denominadas no estacionarias y se caracterizan por poseer media y varianza no constantes a lo largo del tiempo. Para comprobar la diferencia entre las series estacionarias y las no estacionarias, consideremos la siguiente caracterización de $\boldsymbol{y}$.

$$
y_{t}=\beta+y_{t-1}+e_{t}
$$

en la cual, a diferencia de la representada en (3), se considera que la variable está determinada por los valores anteriores de ella misma. Sustituyendo recursivamente y suponiendo un nivel inicial de la variable, $y_{0}$, llegamos a la expresión:

$$
y_{t}=y_{0}+\beta t+\sum_{j=0}^{t} e_{j}
$$

cuya diferencia con (3) se encuentra en el último término. En (5) éste se interpreta como el efecto en el período actual de los shocks ocurridos en el pasado, representados por el término de perturbación e. Sin embargo, como se puede deducir, si e presenta raíces unitarias, los shocks tienen un efecto permanente sobre los valores de la serie. Por el contrario, en (3) el efecto sobre el producto de la variable estocástica $\boldsymbol{u}$ es transitorio. Es posible, por tanto, distinguir dos modelos para $\boldsymbol{y}_{\boldsymbol{t}}$. En el primer, estacionario respecto a una tendencia $t$, el efecto de los shocks exógenos es transitorio. En el segundo, estacionario en diferencias, su efecto es permanente.

Como se acaba de indicar, estos trabajos confirmaron que la mayoría de las series macroeconómicas eran no estacionarias. Por tanto, las alteraciones experimentadas por las series en el pasado persistían en el presente. La implicación de ello suponía que en estas series era difícil distinguir los cambios que podemos denominar «grandes shocks», infrecuentes y con efectos sobre la tendencia o el nivel de la serie de forma permanente, de los «bocks corrientes» (o habituales), que ocurririan de forma regular y podrían o no tener efectos permanentes sobre el nivel de las series, de acuerdo con la hipótesis de la raíz unitaria.

Los grandes shocks son los que mayor interés pueden tener para la historia económica, por cuanto su existencia implica un cambio en el comportamiento de las variables. Éstos pueden subdividirse en tres grupos: 
a) cambio de nivel, en los que la variable aumenta (o disminuye) en el año posterior a la ruptura y su crecimiento se reanuda a la misma tasa que antes de la misma; $b$ ) cambios en la tasa de crecimiento, cuando la tasa de crecimiento es mayor (o menor) a partir del año siguiente a la ruptura, y c) cambios conjuntos de nivel y tasa de crecimiento. Su representación, en sentido creciente, figura en el gráfico 1.

\section{GRÁFICO 1}

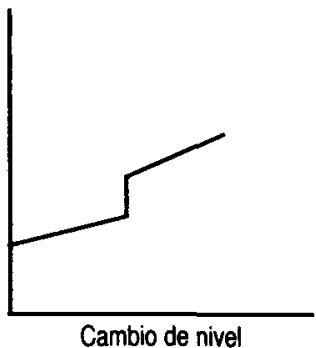

Cambio de nivel

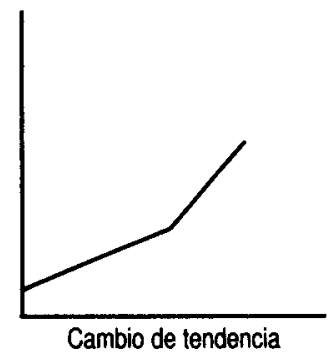

Cambio de tendencia

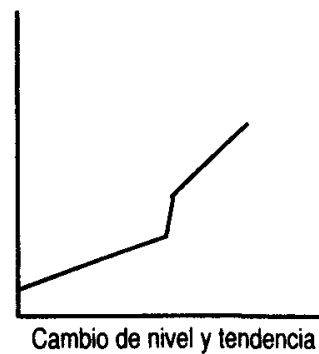

El método para detectar la presencia de un cambio estructural consiste en realizar la regresión para los diferentes modelos de ruptura de acuerdo con la especificación elegida y comprobar la hipótesis nula de presencia de una raíz unitaria, es decir, contrastar la persistencia temporal del shock analizado. Una diferencia entre la metodología empleada por Perron y la empleada aquí es el carácter de la ruptura. Como hemos mencionado, Perron determinaba exógenamente el punto de ruptura, es decir, suponía que la evolución de la serie no generaba la quiebra en su comportamiento, sino que ésta era externa a la serie. El efecto de actuar de esta manera es que los puntos de ruptura analizados son aquellos en donde parece más evidente el cambio de comportamiento de las series. Por tanto, los estadísticos derivados estaban sesgados a favor de rechazar la hipótesis nula de ausencia de ruptura. La estrategia seguida por Zivot y Andrews $(1992)^{8}$ fue desarrollar un proceso para determinar los puntos de ruptura y derivar los estadísticos apropiados para esta nueva situación. Su solución consiste en la construcción de un algoritmo para obtener el punto de ruptura que otorgue mayor probabilidad a la existencia de un corte en la serie. La argumentación podría

${ }^{8}$ Una aproximación similar fue desarrollada independientemente por Christiano (1992), pp. 237-250. 
resumirse de forma simplificada en la definición de la hipótesis nula para cada uno de los tres modelos como un proceso integrado de orden uno:

$$
y_{t}=\mu+y_{t-1}+e_{t}
$$

frente a una hipótesis alternativa representada por un modelo estacionario en torno a una tendencia con un único punto de ruptura. Los modelos a estimar anidan la hipótesis nula y alternativa:

$$
\begin{gathered}
y_{t}=\mu^{A}+\theta^{A} D U_{t}+\beta^{A} t+\alpha^{A} y_{t-1}+\sum_{i=1}^{k} c_{i} \Delta y_{t-i}+e_{t} \\
y_{t}=\mu^{B}+\beta^{B} t+\gamma^{B} D T^{*}+\alpha^{B} y_{t-1}+\sum_{i=1}^{k} c_{i} \Delta y_{t-i}+e_{t} \\
y_{t}=\mu^{C}+\theta^{C} D U_{t}+\beta^{C} t+\gamma^{C} D T+\alpha^{C} y_{t-1}+\sum_{i=1}^{k} c_{i} \Delta y_{t-i}+e_{t}
\end{gathered}
$$

donde $D U_{t}=1$ si $t>T_{B}$ y 0 en otro caso, $T_{B}$ es el año de ruptura; $\mathrm{DT}_{\mathrm{t}}{ }^{*}=\mathrm{t}-\mathrm{T}_{\mathrm{B}}$ si $\mathrm{t}>\mathrm{T}_{\mathrm{B}}$ y 0 en otro caso y donde $\mathrm{DT}_{\mathrm{t}}=\mathrm{t}$ si $\mathrm{t}>\mathrm{T}_{\mathrm{B}} \mathrm{y}$ 0 en otro caso. Los $k$ regresores añadidos en cada ecuación eliminan comportamientos distorsionadores en el componente estocástico. Su número ha sido determinado mediante test de significación de los coeficientes estimados, $\boldsymbol{c}_{\boldsymbol{i}}$.

Por tanto, la metodología empleada a continuación consiste en realizar la regresión correspondiente al modelo elegido en un rango que incluya todo el período analizado. El objetivo es permitir la libre variación del momento de ruptura a lo largo de toda la serie, eligiendo la observación en la que la probabilidad de aceptarla es máxima. Esto se consigue eligiendo el momento del tiempo en el cual la significatividad del parámetro de la variable endógena retardada es máximo, es decir, cuando la significación de la presencia de una raíz unitaria en la serie es mayor.

En conclusión, la estrategia para hallar la existencia de una discontinuidad en el comportamiento de la serie analizada consiste en: i) comprobar la existencia de raíz unitaria, como medio de conocer la persistencia de los shocke; ii) realizar la regresión del modelo de ruptura que más se acerque al comportamiento de la serie o analizar los tres modelos, suponiendo que el período en el que tiene lugar la ruptura varía desde el inicio de la serie hasta el final de la misma; iii) hallar el valor del estadístico $t$ del coeficiente de la variable endógena retardada para cada período de ruptura calculado, y iv) comparar el valor máximo del estadístico $t$ con los valores tabulados. 


\section{CONTINUIDAD Y RUPTURA EN EL CRECIMIENTO ECONÓMICO ESPAÑOL}

Descrito el método, el objetivo ahora es presentar los resultados para comprobar si éstos permiten identificar una discontinuidad en el comportamiento de las diferentes series representativas del crecimiento económico español. Siguiendo el trabajo de Dickey y Fuller, el test para el cálculo de raíces unitarias toma la forma de la regresión:

$$
y_{t}=\mu+\beta t+\alpha y_{t-1}+\sum_{i=1}^{k} c_{i} \Delta y_{t-i}+e_{t}
$$

El cuadro 1 presenta los valores del estadístico t del coeficiente $\alpha$ para las diferentes series, las cuales han sido previamente transformadas en logaritmos. La primera columna muestra los valores de la regresión realizada en niveles y la segunda en diferencias. Como puede comprobarse, no es posible rechazar la presencia de raíces unitarias en la serie de inversión pública y el PNB. La serie de inversión privada rechaza la existencia de raíces unitarias al 5 por 100 , sin embargo, empleando el test de Phillips-Perron no es posible mantener este rechazo. La serie de Índice de Producción Industrial rechaza la existencia de raíz unitaria al 5 por 100 , tanto utilizando el procedimiento Dickey-Fuller ampliado como el Phillips-Perron. En todos los casos, la estimación en primeras diferencias rechaza la existencia de raíces unitarias.

Los resultados obtenidos, por tanto, apuntan a la presencia de raíces unitarias en todas las series, esto es, a la existencia de shocks cuyos efectos se han mantenido a lo largo del tiempo en todas ellas, a lo que aporta evidencia adicional la observación de los gráficos de las series, representados en las páginas que siguen. Como hemos señalado, nuestro interés es separar aquellos cambios profundos y persistentes de los movimientos leves que son reabsorbidos por la misma evolución de las variables y analizar si los primeros están provocados por la presencia de discontinuidades en la trayectoria seguida por cada serie.

Parece obvio que la primera a analizar debe ser la del PNB elaborada por Leandro Prados de la Escosura. Aun cuando sus datos están sujetos a su presentación definitiva, la estimación, como ya hemos indicado, ha sido ampliamente utilizada por la generosidad de su autor. El gráfico 2 representa su comportamiento entre 1850 y 1935, una etapa durante la cual la historiografía ha apuntado la existencia de tres grandes discontinuidades respecto a las décadas precedentes: i) los años de librecambio moderado iniciados con la aprobación del Arancel de 1869; 


\section{CUADRO 1}

Test de raices unitarias, 1850-1935

\begin{tabular}{|c|c|c|}
\hline & $y_{t}$ & $D y_{t}$ \\
\hline 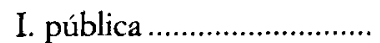 & $-2,22(0)$ & $-5,53^{b}$ \\
\hline I. privada & $-3,74^{a}(1)$ & $-4,81^{b}$ \\
\hline Phillips-Perron .................... & $-3,38$ & $-6,77^{\mathrm{b}}$ \\
\hline IPIES & $-3,60^{\mathrm{a}}(2)$ & $-6,64^{b}$ \\
\hline Phillips-Perron .................. & $-3,52^{\mathrm{a}}$ & $-10,17^{b}$ \\
\hline PNB & $-2,34(0)$ & $-5,63^{b}$ \\
\hline
\end{tabular}

NOTA: Entre paréntesis el número de retardos de la variable endógena necesarios para eliminar la autocorrelación. Los valores críticos son 3,46 al 5 por 100 y 4,07 al 1 por $100 .^{a}$ significa rechazo de la hipótesis nula de raíz unitaria al 5 por $100 ;{ }^{b}$ significa rechazo de la hipótesis nula de raíz unitaria al 1 por 100 .

ii) la elevación de los aranceles en 1891, 1906 y 1922 y el abandono del patrón oro en la década de los ochenta, y más claramente en 1891, y iii) la Primera Guerra Mundial.

El cuadro 2 y los gráficos $\mathrm{A}$ del apéndice recogen el análisis de ruptura empleando las ecuaciones 7, 8 y 9 para buscar el menor (mayor en términos absolutos) valor del estadístico $t$ a lo largo de toda la muestra. En todos los modelos utilizados los valores más bajos del estadístico se obtienen en los años veinte. En el modelo de cambio de nivel aparecen dos picos: uno en torno a 1885 y otro en 1919. Al tratar el cambio de tendencia (véase el apéndice), los valores de $t$ del coeficiente asociado a la variable endógena retardada aumentan continuamente hasta alcanzar el máximo en el último de los años mencionados. En el modelo de cambio de nivel y tendencia aparecen diversos puntos de posible ruptura, encontrándose el máximo en 1914. Sin embargo, y a pesar de esta evidencia coincidente con las tesis mantenidas habitualmente sobre la alteración de las pautas del crecimiento a partir de la Primera Guerra Mundial, no se puede aceptar la existencia de una ruptura, en el sentido definido en estas páginas, en ningún período con una significatividad del 5 por 100 empleando los valores críticos tabulados por Zivot y Andrews 9 .

${ }^{9}$ Perron y Vogelsgan (1992) sugieren que en los cambios de nivel la estimación de la ecuación (7) puede no ser adecuada, siendo más apropiado estimar:

$$
y_{t}=\mu^{A}+\theta^{A} D U_{t}+\beta^{A} t+d^{A} D(T B)_{t}+\alpha^{A} y_{t-1}+\sum_{i=1}^{k} c_{i} \Delta y_{t-i}+e_{t}
$$




\section{GRÁFICO 2}

Logaritmo del PNB, 1850-1935

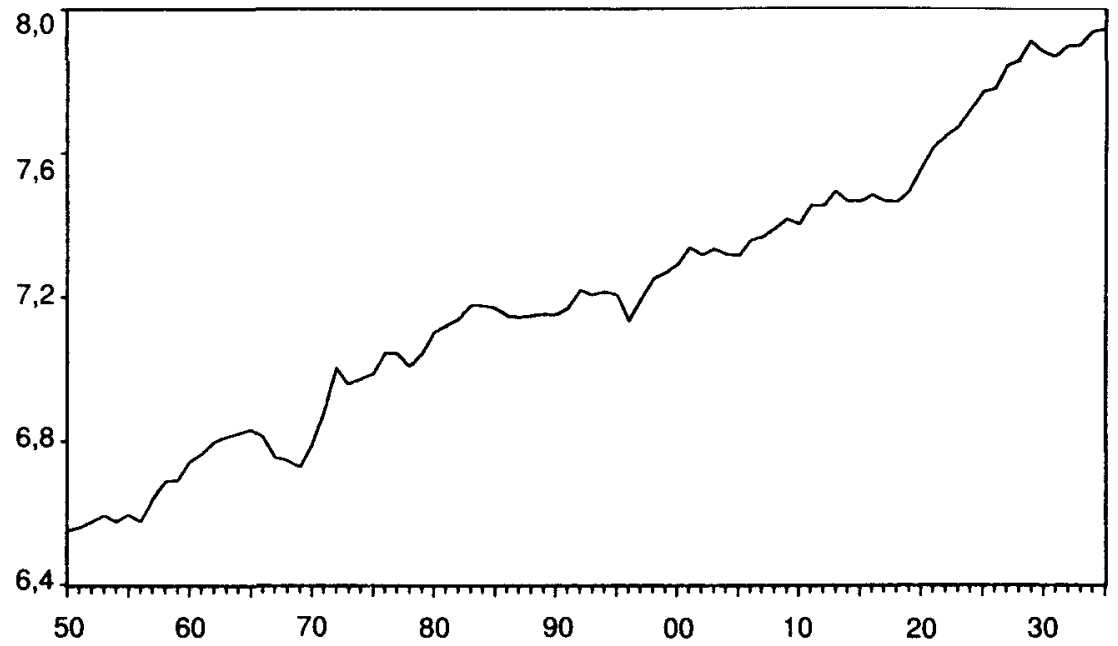

CUADRO 2

Discontinuidades en el PNB, 1850-1935

\begin{tabular}{|c|c|c|c|}
\hline & Nivel & Tendencia & Ambos \\
\hline 1870 & $-2,15$ & $-2,05$ & $-2,15$ \\
\hline $1891 \ldots$ & $-2,89$ & $-2,22$ & $-3,11$ \\
\hline 1919 & $-3,36$ & $-2,84$ & $-2,96$ \\
\hline
\end{tabular}

Por tanto, de corroborarse en la versión definitiva la información cuantitativa ofrecida por la estimación actualmente disponible del PNB, la caracterización más adecuada de su evolución en el largo plazo es la

que incluye una variable adicional, $D(T B)_{t}$, que toma el valor 1 en el año de ruptura y cero en cualquier otro momento del tiempo. Sin embargo, esta estimación no altera los resultados. Nuevamente el valor máximo del estadístico $t$ (en valor absoluto), $-3,41$, se alcanza en 1919, con dos retardos en las diferencias de la variable endógena, pero continúa siendo inferior al valor crítico tabulado por Perron y Vogelsgan de $-4,39$ para una significatividad del 5 por 100. Suponiendo que los puntos de ruptura se determinan exógenamente Cratfs y Mill encuentran un cambio estadísticamente significativo en la tasa de crecimiento del producto per cápita español en 1914. [Cfr. Crafts y Mill (1996), pp. 415-431.] 
de un crecimiento continuado durante cerca de un siglo, sin experimentar una súbita etapa de ruptura en ninguna de las tres coyunturas habitualmente subrayadas por la historiografía ni tampoco en el cambio de siglo. A pesar de que los resultados apuntan a que a partir de los años de la Primera Guerra Mundial tienen lugar cambios muy destacados en las funciones de producción, no es posible aceptar la existencia de una discontinuidad estadísticamente significativa. De acuerdo con ello, puede afirmarse que el comportamiento del PNB, según la estimación realizada por Prados de la Escosura, es el resultado tanto de la tendencia como de la acumulación de los efectos de los diferentes shocks producidos a lo largo del tiempo. La conclusión a deducir es inmediata y parece relevante. El contraste realizado descarta que tanto los cambios en la política económica o las relaciones con el exterior (del librecambio moderado al proteccionismo y de una vinculación con los países del patrón-oro a un aislamiento en las relaciones financieras internacionales), como las transformaciones económicas que cristalizan a partir de Primera Guerra Mundial modificaran estructuralmente el comportamiento a largo plazo de la economía.

La presencia dentro de ésta de un importante sector agrario cuya evolución era en buena medida independiente de la marcha del resto de la economía por la influencia de la calidad de la tierra y los factores climáticos invita a considerar si frente a este resultado en la trayectoria del PNB, la evolución del Índice de Producción Industrial (IPIES) presenta discontinuidades estructurales. La evolución de esta variable ha sido sometida a un minucioso escrutinio en busca de las raíces del aumento del tamaño del sector secundario. La discusión se ha centrado, en buena medida, en el papel desempeñado por la política protectora establecida en la década final del siglo Xxx y en la inicial del siglo $\mathrm{XX}$, así como en el efecto de la Primera Guerra Mundial y la Dictadura de Primo de Rivera, aun cuando, con una insistencia creciente, se abre paso la hipótesis de un cambio en los parámetros tecnológicos de los sectores industriales.

Aportaciones realizadas antes de la construcción del Índice de Producción Industrial [Donges (1975) y Fontana y Nadal (1975)] apuntaron que la industrialización española arrancaba a principios de este siglo, favorecida por la adopción de fuertes aranceles protectores y de medidas específicas de fomento de la industria nacional. En clara contraposición con esta tesis, el autor de la estimación, Albert Carreras, ha escrito que «no existe discontinuidad alguna en el crecimiento industrial en torno a 1900 , ni en torno a 1890 ó 1906. (...) Muy al contrario: a la vista de los nuevos datos parece más legítimo considerar que lo que 
hay en los noventa años anteriores a la Guerra Civil (...) es un largo período de crecimiento, unas veces algo más rápido, otras algo más lento, que da a entender que la tradición industrializadora es larga en España»10. Esta continuidad durante el período de entreguerras, sin embargo, no implica ignorar que durante esos años, la industria experimentó un ritmo de crecimiento «espectacular» ${ }^{11}$ simultáneo a una intensa modernización y diversificación ${ }^{12}$ de su estructura productiva.

El gráfico 3 presenta la evolución del Índice de Producción Industrial y el cuadro 3 los valores del estadístico $t$ para los tres modelos. En los dos momentos con menor valor del estadístico (1891 y 1921) no puede aceptarse la hipótesis de cambio estructural. Ello confirma la tesis planteada por Carreras frente a los defensores de la aceleración del cambio en cualquiera de las coyunturas apuntadas y, junto a los resultados obtenidos en el caso del PNB, refuerza una interpretación de la trayectoria de la economía en este largo período entre 1850 y 1936, basada más en la continuidad que en una ruptura vinculable a la existencia de un «brote industrializador» gerschenkroniano $o$, si se prefiere la terminología de Rostow, del inicio de un despegue. Aun sin negar las repercusiones de la nueva frontera de posibilidades tecnológicas abiertas por la difusión de la electricidad, su reflejo en la serie muestra que éstas no fueron de una magnitud suficiente para modificar los patrones fundamentales del crecimiento del producto industrial.

Junto a esta conclusión, los resultados muestran una discontinuidad estadísticamente significativa en el año 1870. Como se observa en los gráficos $\mathrm{B}$ del Apéndice, al emplear el modelo de cambio de nivel, el valor absoluto más elevado del estadístico t se encuentra en esa fecha, con un valor de $-4,95$ inferior al valor crítico significativo al 5 por 100 de $-4,80$. La metodología de Perron y Vogelsang confirma el resultado, y refuerza la tesis de la existencia de un cambio de este tipo en ese año. Las implicaciones de este resultado no son fáciles de realizar por cuanto hasta este momento no ha sido subrayado. Parece evidente, sin embargo, que esta alteración supone una confirmación de las positivas consecuencias de la política económica liberal del Sexenio sobre el sector industrial, dentro de la cual sobresale, aun cuando no la agote, la reforma arancelaria de Figuerola ${ }^{13}$.

${ }^{10}$ Carreras (1987), p. 284.

11 Carreras (1987), p. 288.

${ }^{12}$ Betrán (1995), cap. III, pp. 133-206.

${ }^{13}$ La mejor síntesis de la política económica del Sexenio sigue siendo la de Tortella (1975), cap. VII, pp. 293-318. 


\section{GRÁFICO 3}

Logaritmo del IPI, 1850-1913

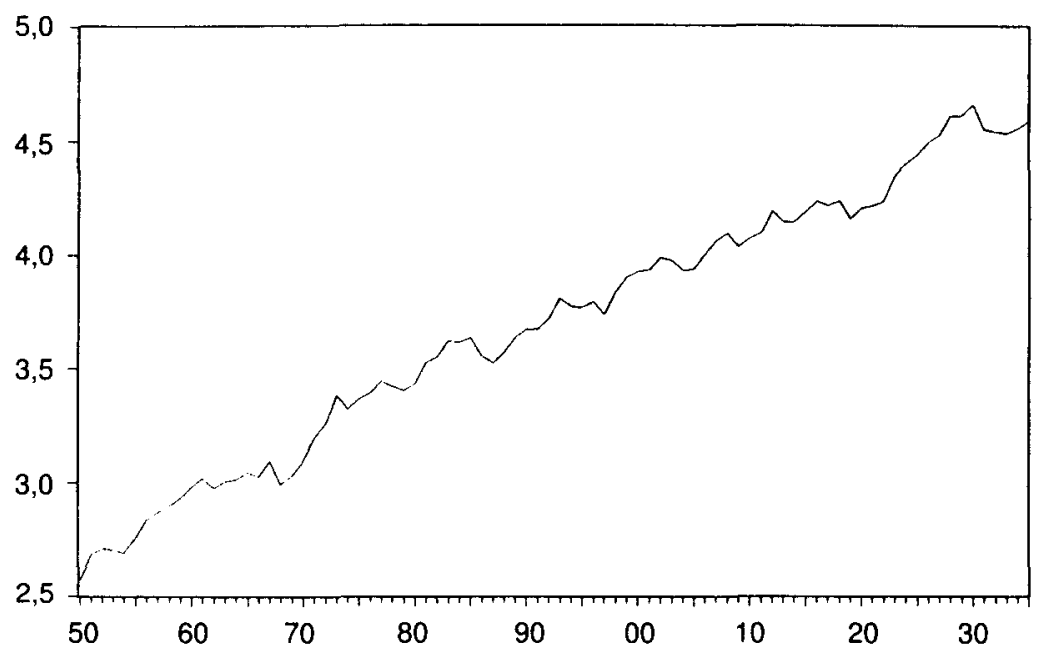

CUADRO 3

Discontinuidades en el IPI, 1850-1935

\begin{tabular}{llcc}
\hline & Nivel & Tendencia & Ambos \\
\cline { 2 - 4 } $1891 \ldots \ldots \ldots \ldots \ldots . .$. & $-4,11$ & $-3,75$ & $-3,95$ \\
$1921 \ldots \ldots \ldots \ldots \ldots \ldots . .$. & $-3,35$ & $-3,34$ & $-3,62$ \\
\hline
\end{tabular}

Además de la evolución del producto, tiene interés contrastar en qué medida la trayectoria seguida por la inversión, tanto privada como pública, presenta discontinuidades ${ }^{14}$. El comportamiento de la inversión privada en el largo plazo ha sido descrito como «una fluctuación errática en torno a unos niveles estables» ${ }^{15}$. Y en su observación gráfica se aprecia un mayor componente cíclico que en las series del PNB y del IPIES. Los contrastes de cambio estructural, sin embargo, ofrecen también resultados negativos. Como puede comprobarse en los gráficos $\mathrm{C}$ del apéndice los estadísticos

${ }^{14}$ En todos los casos, la referencia en estas páginas a rupturas o discontinuidades se refiere a aquellas estadísticamente significativas.

is Carreras (1985), p. 31. 
no alcanzan los valores críticos en ningún momento, encontrándose el máximo en 1855 . Una observación de la serie original muestra que esa fecha se corresponde con el punto de inicio de un ciclo inversor asociado a la construcción de la red ferroviaria. El ejercicio realizado, pues, vendría a confirmar dos aspectos de interés. Por un lado, la continuidad -ya mencionada en el caso del PNB y del IPIES - como rasgo dominante del crecimiento español entre 1855 y 1936. Por otra, la importancia de la coyuntura del Bienio Progresista en la tendencia seguida por la inversión, una variable fundamental de todo proceso de crecimiento, sin que, al igual que sucede con la coyuntura extraordinaria de la Primera Guerra Mundial, sus repercusiones fueran suficientes para provocar una ruptura en la serie.

\section{GRÁFICO 4}

Logaritmo de la inversión privada, 1850-1935

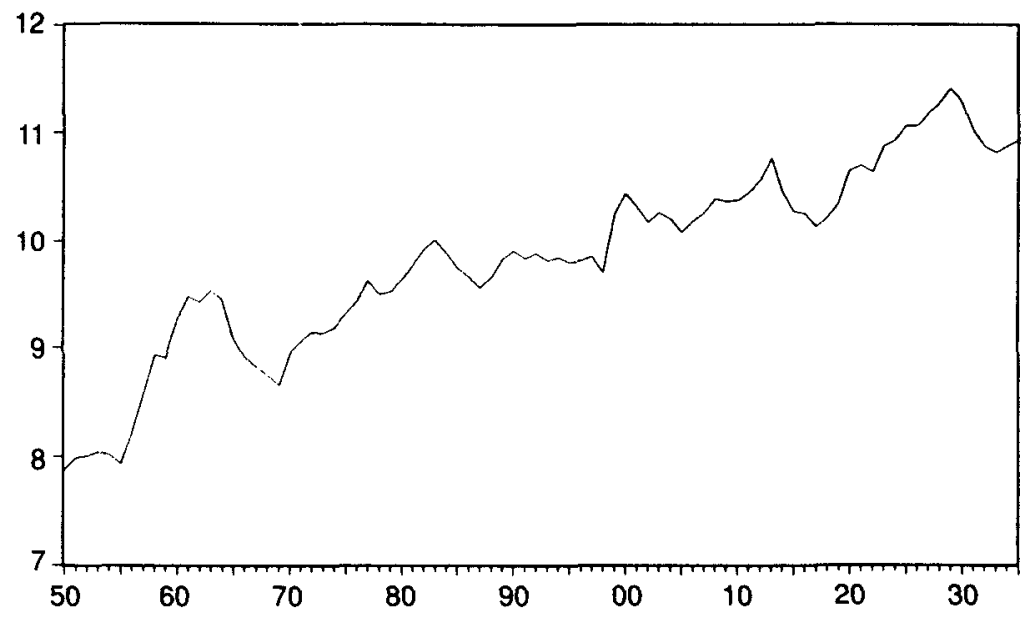

Más controvertido ha resultado la descripción y el análisis de la evolución de la inversión pública. Tedde de Lorca y Comín han distinguido entre un Estado liberal, cuya intervención en la economía era poco relevante como consecuencia de la escasez de fondos obtenidos por un sistema fiscal siempre insuficiente, y un Estado más intervencionista en asuntos económicos y sociales, con la transición entre ambos en las dos primeras décadas del siglo $\mathrm{xx}^{16}$. Palafox y Cubel, por su parte, han subrayado la

${ }^{16}$ Comín (1988), p. 644, y Tedde de Lorca (1981), pp 237-265, y (1996), pp. 16-49. 


\section{CUADRO 4}

Discontinuidades en la inversión privada, 1850-1935

\begin{tabular}{lccc}
\hline & Nivel & Tendencia & Ambos \\
\cline { 2 - 4 } $1855 \ldots \ldots \ldots \ldots \ldots . \ldots$. & $-3,88$ & $-3,36$ & $-3,87$ \\
$1869 \ldots \ldots \ldots \ldots \ldots$. & $-2,93$ & $-2,61$ & $-2,15$ \\
$1890 \ldots \ldots \ldots \ldots \ldots$. & $-2,85$ & $-2,75$ & $-2,77$ \\
$1922 \ldots \ldots \ldots \ldots \ldots$. & $-2,85$ & $-2,83$ & $-3,11$ \\
\hline
\end{tabular}

importancia del cambio experimentado por la función inversora del Estado tras la I Guerra Mundial, cuando se produjo un incremento en la participación de la inversión pública en el PNB hasta duplicar los valores alcanzados en las décadas previas ${ }^{17}$ con un impacto positivo y significativo en la trayectoria de la economía tanto mayor cuando más se avanza en el primer tercio del siglo ${ }^{18}$.

Frente a los resultados de los contrastes realizados hasta aquí, en donde, excepto el cambio de nivel en el Índice de Producción Industrial en 1870, la continuidad es la norma, el análisis de la serie de inversión pública muestra resultados muy distintos. Éstos permiten identificar una alteración significativa de su comportamiento en los años veinte. Los gráficos $\mathrm{D}$ del apéndice presentan la evolución del estadístico $t$ junto con la línea discontinua que refleja el valor crítico para cada modelo. En las tres especificaciones se produce un aumento del valor absoluto del estadístico en 1919, llegando a superar el valor crítico en el modelo que permite un cambio en la media de la serie. En consecuencia, según la técnica utilizada, existe una ruptura en el comportamiento de la inversión pública en ese año consistente en un aumento de su nivel sin aumento en el ritmo de crecimiento ${ }^{19}$. Por tanto, independientemente de la controversia sobre cuál fue su impacto macroeconómico, queda confirmado que, a partir de 1919, se altera el comportamiento de la acción inversora del sector público.

17 Palafox y Cubel (1996), p. 116.

18 Palafox (1991), en especial nota IV, pp. 316-319, y Cubel (1993), pp. 49-77.

${ }^{19}$ Como en ocasiones anteriores se ha realizado la misma estimación con las especificaciones recomendadas por Perron y Vogelsang aceptándose la hipótesis de ruptura en la serie con un nivel de significatividad del 2,5 por 100 . 


\section{GRÁFICO 5}

Logaritmo de la inversión pública, 1850-1935

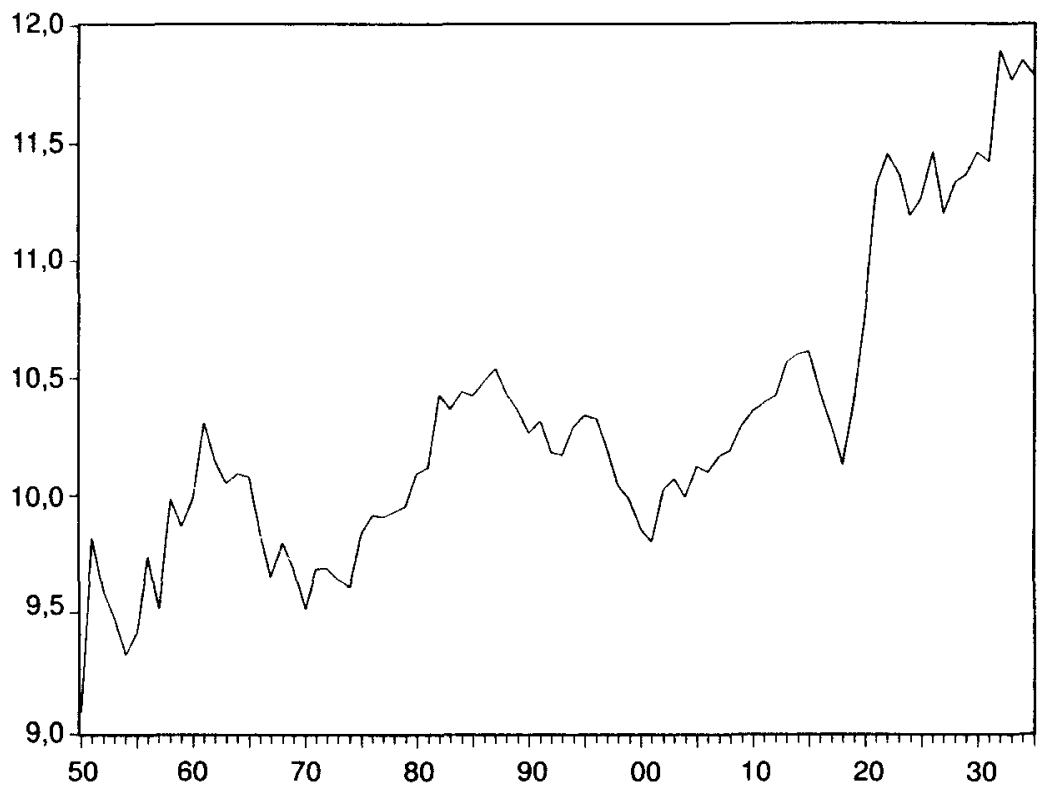

CUADRO 5

Discontinuidades en la inversión pública, 1850-1935

\begin{tabular}{lccc}
\hline & Nivel & Tendencia & Ambos \\
\cline { 2 - 4 } $1896 \ldots \ldots \ldots \ldots \ldots . .$. & $-2,44$ & $-3,37$ & $-4,51$ \\
$1919 \ldots \ldots \ldots \ldots \ldots \ldots . .$. & $-4,85$ & $-3,51$ & $-4,60$ \\
\hline
\end{tabular}

\section{CONCLUSIONES}

Como se ha indicado al comienzo, el objetivo de estas páginas es aprovechar los avances en el acervo cuantitativo de la historia de la economía española antes de 1936 para contrastar diferentes hipótesis de cambio estructural en el comportamiento de las series más relevantes utilizadas por los historiadores de la economía española. Antes de resumir muy brevemente las conclusiones ya apuntadas a lo largo de las páginas 
precedentes no es posible dejar de reiterar las cautelas esbozadas entonces. Teniendo en cuenta la evidencia, sin duda más dispersa pero no menos significativa, en la que se basan las tesis defendidas en la literatura, y las destacadas, y quizá insalvables, dificultades de recopilación de información cuantitativa, no puede descartarse la existencia de deficiencias apreciables en los datos relativos al siglo XIX que podrían impedir la detección de posibles discontinuidades y, por consiguiente, la obtención de conclusiones diferentes. Por otro lado, no es posible dejar de insistir de nuevo en que las discontinuidades a las que se refieren los contrastes realizados están referidas al concepto adoptado en la literatura econométrica más restringido que en el utilizado en la economía del desarrollo o la propia historia económica; esto es, un cambio en los parámetros de la regresión y no a la forma en que opera la economía ${ }^{20}$.

Aun subrayando esta cautela, la información cuantitativa acumulada en los últimos años es, en nuestra opinión, lo suficientemente sólida para subrayar el resultado más destacado de los contrastes realizados: la confirmación de la continuidad, en la que Carreras ha insistido reiteradamente, del crecimiento desde 1850 hasta 1935, sin la presencia de períodos en los que se pueda situarse un «impulso industrializador» específico. La única, aun cuando relevante, excepción, y ésta sería una segunda conclusión a destacar, es la inversión pública. En este caso sí se constata una clara discontinuidad, consecuencia de un bien documentado cambio hacia un sector público más intervencionista y corporativo.

Por otro lado, y en tercer lugar, las series de inversión privada y el Índice de Producción Industrial no muestran rupturas en el largo plazo a excepción del cambio de nivel aparecido en este último en 1870. Éste es un resultado no destacado hasta ahora, vinculable directamente a la metodología utilizada, y vendría a confirmar la trascendencia notable para el crecimiento industrial de las medidas liberalizadoras del Sexenio. Por último, considerando conjuntamente los resultados sobre las series de inversión pública y de $\mathrm{PNB}$, aun cuando en este último caso no se alcancen los valores críticos, parece posible plantear que los años de la I Guerra Mundial son donde se acumulan un número mayor de evidencias de alteración de las pautas de evolución de la economía española ${ }^{21}$, ya señaladas en la utilización de esta metodología, pero suponiendo rupturas determinadas exógenamente, por parte de Crafts y Mill. Pero el conjunto

${ }^{20}$ Maddala y Kim (1996), p. 82.

${ }^{21}$ Es también a partir de entonces cuando el stock de capital físico aumenta de forma destacada. Cfr. Cubel y Palafox (1997), pp. 132-133. 
de contrastes efectuados aquí no puede llevar a ignorar la conclusión principal mencionada: la evidencia cuantitativa hoy disponible corrobora la tesis de una continuidad básica en el crecimiento económico español durante la dilatada etapa transcurrida entre 1850 y 1936. Dentro de ella, las discontinuidades que sobresalen son dos cambios de nivel: el de la producción industrial en 1870 y el de la inversión pública en 1919. Lo cual, parece evidente, plantea no pocos interrogantes acerca del complejo y dilatado proceso que ha conducido en España a la sociedad industrializada. 


\section{APÉNDICE}

Gráficos A. Discontinuidades en el PNB, 1850-1935

Modelo de cambio de nivel (Valor crítico al 5 por $100,-4,80$ )

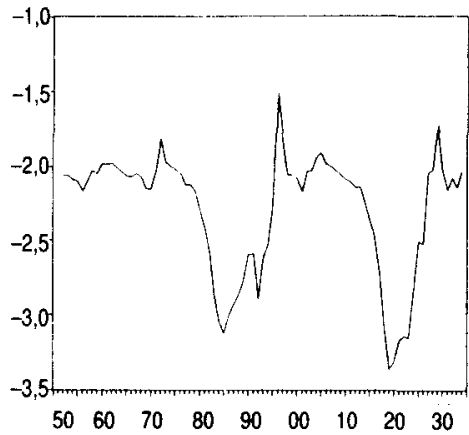

Modelo de cambio de tendencia (Valor crítico al 5 por 100,-4,42)

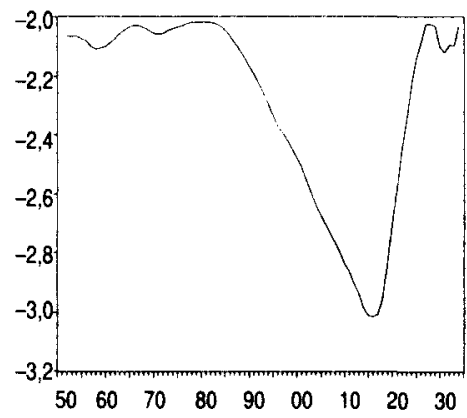

Modelo de cambio de nivel y tendencia (Valor crítico al 5 por $100,-5,08$ )

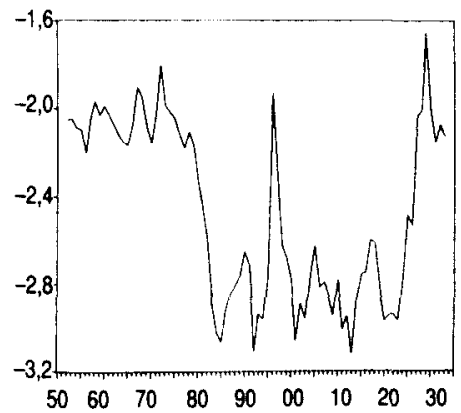

Gráficos B. Discontinuidades en el IPI, 1850-1935

Modelo de cambio de nivel (Valor crítico al 5 por $100,-4,80$ )

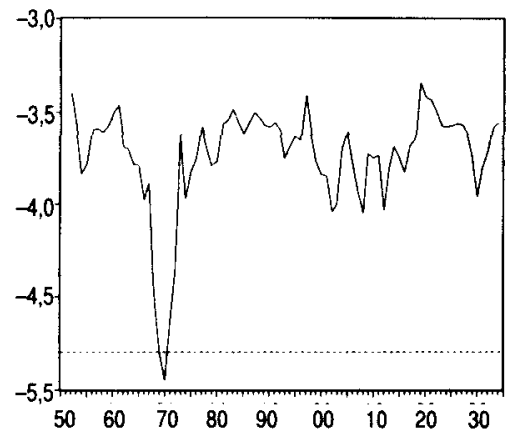

Modelo de cambio de tendencia (Valor crítico al 5 por 100,-4,42)

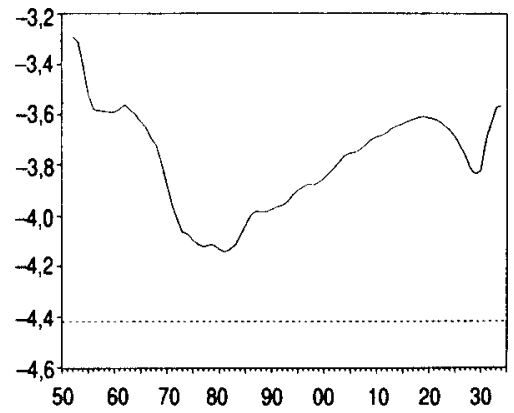

Modelo de cambio de tendencia y nivel (Valor crítico al 5 por $100,-5,08$ )

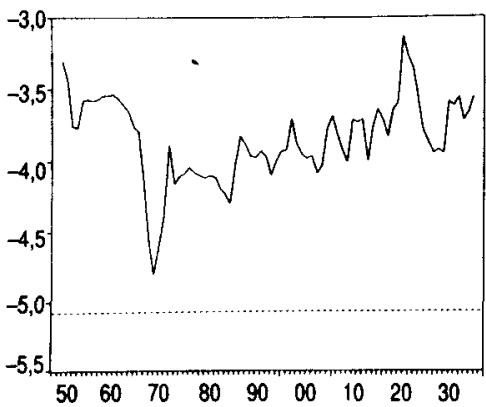


Gráficos C. Discontinuidades

en la inversión privada, $1850-1935$

Modelo de cambio de nivel

(Valor crítico al 5 por $100,-4,80$ )

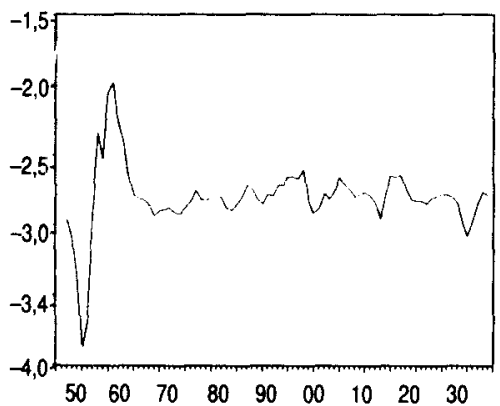

Modelo de cambio de tendencia

(Valor critico al 5 por $100,-4,42$ )

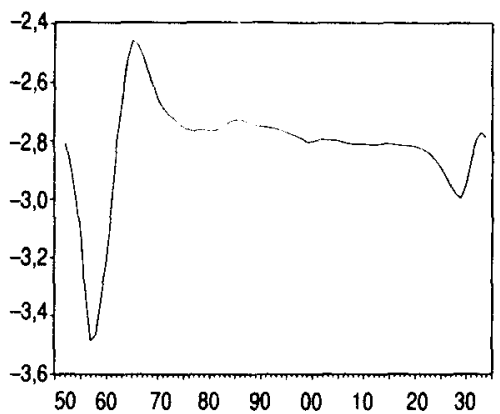

Modelo de cambio de nivel y tendencia

(Valor critico al 5 por $100,-5,08$ )

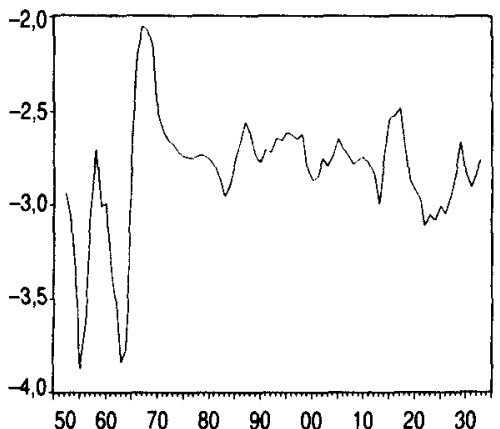

Gráficos D. Discontinuidades

en la inversión pública, 1850-1935

Modelo de cambio de nivel

(Valor crítico al 5 por $100,-4,80$ )

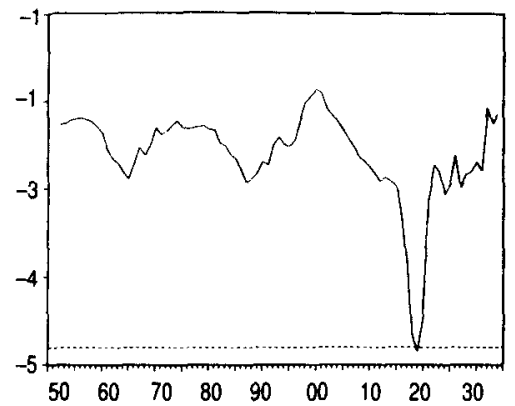

Modelo de cambio de tendencia

(Valor crítico al 5 por $100,-4,42$ )

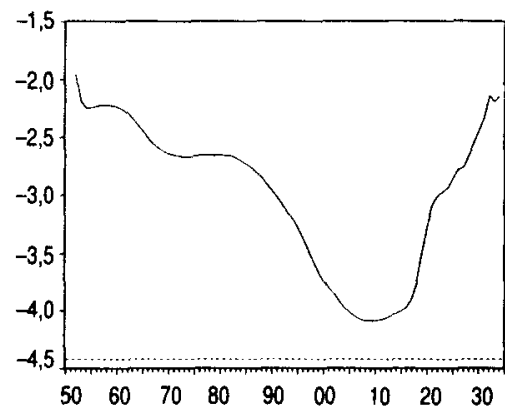

Modelo de cambio de nivel y de tendencia (Valor crítico al 5 por $100,-5,08$ )

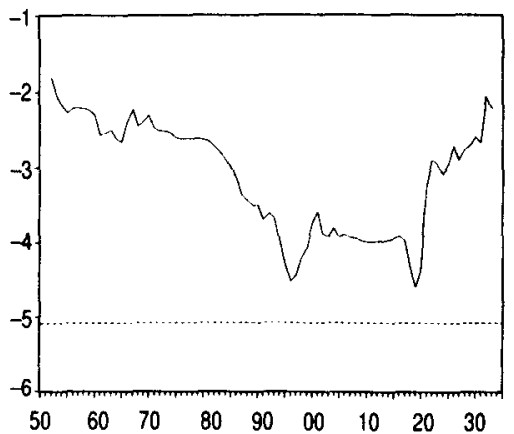




\section{BIBLIOGRAFIA}

Aranzadis, C., y Drazen, A. (1990): «Threshold Externalities in Economic Development», The Quarterly Journal of Economics, vol. CV, núm. 2, pp. 501-526.

Betrán PÉREZ, C. (1995): Crecimiento e industria en el primer tercio del siglo XX. España, 1913-1929, Tesis de doctorado, Universidad de Valencia, mimeo.

CARrerAS, A. (1984): «La producción industrial española, 1842-1941. Construcción de un índice anual», Revista de Historia Económica, II, núm. 1, pp. 127-157.

- (1985): «Gasto Nacional Bruto y formación de capital en España, 1849-1958: primer ensayo de estimación», en MARTín ACENA, P., y Prados de LA Escosura, L. (1985), pp. 17-51.

- (1987): «La industria», en Nadal, J., Carreras, A. y Sudrià C. (comp.), pp. $280-312$.

- (1989) (coord.): Estadisticas Históricas de España, siglos XIX.XX, Madrid, Fundación Banco Exterior.

- (1990): Industrialización española: estudios de bistoria cuantitativa, Madrid, Espasa-Calpe.

Christiano, L. J. (1992): «Searching for a Break in GNP», Joumal of Bussiness and Economic Statistics, vol. 10, núm. 3, pp. 237-250.

Cipolla, C. (1975): The Fontana Economic History of Europe, Londres, Collins Fontana. [Trad. cast. (1980): Historia Económica de Europa, vol. 6.2 , Barcelona, Ariel.]

Comin, F. (1988): Hacienda y economia en la España contemporánea, 1800-1936, Madrid, Instituto de Estudios Fiscales.

Crafts, N., y Harley, C. (1992): «Output Growth and the British Industrial Revolution: a restatement of the Crafts-Harley view», Economic History Review, vol. XIV, pp. 703-730.

Crafts, N. F. R., y Mill, T. C. (1994): «The Industrial Revolution as a Macroeconomic Epoch: an alternative view», Economic History Review, vol. XLVII, núm. 4, pp. 769-775.

- (1996): «Europe's Golden Age: an econometric investigation of changing trend rates of growth», en VAN ARK, B. y CRAFTs, N. (1996), pp. 415-431.

Cubel, A.,(1993): «Gasto Público y crecimiento económico en la Restauración (1874-1923)», Revista de Historia Económica, XI, núm. 1, pp. 49-77.

- y PALAFOX, J. (1997): «El stock de capital de la economía española. 1900-1958», Revista de Historia Industrial, núm. 12, pp. 113-146.

Donges, J. B. (1976): La industrialización en España, Barcelona, Oikos-Tau.

FISHLOW, A. (1965): «Empty Economic Stages», Economic Joumal, vol. LXXV, marzo, pp. 112-125.

Fontana, J., y Nadal, J. (1975): «Spain, 1914-1970», en Cipolla, C. (1975), vol. 6.2, pp. 460-529.

Gerschenkron, A. (1968): Continuity in History and Other Essays, Cambridge, The Belknap Press of Harvard University Press.

Greasley, D., y OxIEY, L. (1994): «Rehabilitation Sustained: the Industrial Revolution as a Macreconomic Epoch», Economic History Review, vol. XIVII, núm. 4, pp. 760-768. 
- (1996): «Discontinuities in Competitiveness: the Impact of the First World Wat on British Industry», Economic History Review, vol. XIIX, núm. 1, pp. 82-100.

Gómez Mendoza, A. (1995): Los obstáculos físicos al desarrollo de la industria, Fundación Duques de Soria, Soria, mimeo.

- (1997): La modernización industrial en España, II Encuentro de Historia Económica, UIMP, Valencia, mimeo.

Herranz, A., y TIRADO, D. (1996): «La restricción exterior al crecimiento económico español (1870-19139)», Revista de Historia Económica, XIV, 1996, núm. 1, pp. 11-49.

InstTtuto DE Estudios Fiscales (1976), Datos básicos para la bistoria financiera de España, 1850-1975, Madrid, Instituto de Estudios Fiscales.

Inwood, K., y Stengos, T. (1991): «Discontinuities in Canadian Economic Growth, 1870-1985», Explorations in Economic History, vol. 28, pp. 274-286.

Kuznets, S. (1963): «Notes on Take-off», en Rostow, W. W. (1963), pp. 53-73.

MADDALA, G. S., y KIM, I. M. (1996): «Structural Change and Unit Roots», Joumal of Statistical Planning and Inference, vol. 49, pp. 73-103.

Martín Aceña, P., y Prados de la Escosura, L. (1985): La Nueva Historia Económica en España, Madrid, Tecnos.

Murphy, K. M.; Shleifer, A., y Vishny, R. (1989): «Industrialization and the Big Push», Journal of Political Economy, vol. 97, núm. 5, pp. 1003-1026.

NADAL, J. (1975): El fracaso de la Revolución industrial en España, 1814-1913, Barcelona, Ariel.

- Carreras, A., y SudriÀ, C. (1987): La economía española en el siglo XX. Una perspectiva bistórica, Barcelona, Ariel.

Nelson, C. R. y Plosser, C. I. (1982): «Trends and Random Walks in Macroeconomic Time Series», Journal of Monetary Economics, vol. 10, pp. 1361-1401.

NiCOLAU, R. (1989): «La población», en A. Carreras (1989), pp. 51-90.

Palafox, J. (1991): Atraso económico y democracia. La Segunda República y la economía española, 1892-1936, Barcelona, Crítica.

- y CuBEL, A. (1996): «El sector público durante el primer tercio del siglo XX», en Ayer, núm. 21, pp. 97-126.

Pérez, F.; Mas, M., y Uriel, E. (1996): El stock de capital en España y sus comunidades autónomas, Madrid, Fundación BBV.

Perron, P. (1989): «The Great Crash, the Oil Price Shock and the Unit Root Hypothesis», Econometrica, vol. 57, núm. 6, pp. 1361-1401.

- y VOGELSGAN, T. J. (1992): «Nonstationarity and level shifts with an application to Purchasing Power Parity», Joumal of Business ans Economic Statistics, vol. 10, núm. 3, pp. 301-320.

Prados de la Escosura, L. (1995): Spain's Gross Domestic Product, 1850-1993: Quantitative Conjectures, Universidad Carlos III, mimeo.

Rostow, W. W. (1960): The Stages of Economic Growth, Cambridge, Cambridge University Press. [Trad. cast. (1961): Las etapas del crecimiento económico. Un manifiesto no comunista, México, Fondo de Cultura Económica.] 
TEDDE DE LORCA, P. (1981): «El gasto público en España, 1875-1906: un análisis comparativo con las economías europeas», Hacienda Pública Española, núm. 69, pp. 237-265.

- (1996): «De la primera a la segunda Restauración. El Sector Público y la modernización de la economía española, 1875-1975», Ayer, núm. 21, pp. 16-49.

TirADO, D. (1996): La protección arancelaria en la Restauración, Tesis doctoral, Universidad de Barcelona, mimeo.

TORTELLA, G. (1973): Los origenes del capitalismo en España, Madrid, Tecnos.

- (1994): El desarrollo de la España contemporánea. Historia económica de los siglos XIX y XX, Madrid, Alianza.

- (1963): The economics of Take-off into Sustained Growth, Londres, Macmillan. [Trad. cast. (1967): La economía del despegue, Madrid, Alianza.]

VAN ARK, B., y CrafTS, N. (eds.): Quantitative Aspects of Post-War European Economic Growth, Cambridge, Cambridge University Press.

ZIVOT, E., y ANDREws, D. (1992): «Further evidence of the Great Crash, the Oil Shock, and the Unit Root Hypothesis», Journal of Business and Economic Statistics, vol. 10, núm. 3, pp. 251-270. 